\title{
Liquid atomization process in medical applications
}

\author{
Tomasz R. Sosnowski, Katarzyna Dobowolska \\ Faculty of Chemical and Process Engineering, Warsaw University of Technology, POLAND, Warsaw, \\ Waryńskiego 1, E-mail: Tomasz.Sosnowski@pw.edu.pl
}

\begin{abstract}
Aerosols are used as carriers of drugs delivered to the lungs by inhalation. One of the common methods of aerosol generation for this purpose is the atomization of liquid medicines in nebulizers. In this work, we present the most important engineering aspects of this process which create a challenge for the efficient and safe administration of pharmaceutics to patients of different ages and health conditions.
\end{abstract}

Keywords - aerosol generation, droplets, nebulization, inhalation, drug delivery.

\section{Introduction}

Drug delivery by inhalation is the most convenient way of therapy for the lung diseases such as asthma, COPD, and others. The treatment requires a simple intake of air with aerosol particles composed of a pharmaceutical agent. Since aerosols are unstable, they must be formed just before the inhalation, and this process is accomplished by inhalers of the various operation principles. If the medicine is available as a liquid, the aerosol can be obtained with the atomization process atomized done in devices known as nebulizers.

Nebulizers produce droplets with the most desirable size (typically smaller than $5 \mu \mathrm{m}$ ) that assures their penetration to the diseased regions of the lungs. Pneumatic (or: jet) nebulizers use compressed air for aerodynamic spraying the liquid into droplets of various sizes. Only the smallest droplets are emitted via the attached mouthpiece or mask, while the larger ones are separated and drain to the bulk liquid. Ultrasonic nebulizers (UN) use the energy of fast acoustic vibrations. The classic UNs atomize liquids via the creation of surface waves (frequency of $\sim 2$ $\mathrm{MHz}$ ) and are not recommended for viscous fluids and suspensions. The so-called vibrating mesh nebulizers eject aerosols from the surface of the porous membrane oscillating with the frequency of 100-200 kHz. Here, the aerosol is less polydisperse, but the droplet size distribution strongly depends on the properties of the mesh and the liquid.

The main advantage of nebulizers is their use during spontaneous breathing, which is very convenient for all groups of patients, including infants or people with disabilities. They may also be used for ventilated patients by attaching the nebulizer into the ventilation circuit.

\section{Selected technical issues of drug atomization in nebulizers}

The problem of reproducible generation of aerosol with the properties optimal for inhalation requires addressing several technical issues:

1) the influence of physicochemical properties of atomized liquid on the aerosol mass output rate and droplet size distribution;

2) the influence of actual inhalation conditions during nebulizer use, i.e., the aerosol flow rate and dilution with the auxiliary air, nebulizer position (angle), the role of attached elements (mouthpiece, inhalation mask, ventilation tubings, inhalation chamber, etc.);

3 ) the influence of technical characteristics of the nebulizer on the aerosol quality (e.g., the droplet size and velocity at the outflow from the device), which determine drug delivery to the defined regions of the respiratory system. 


\section{The available experimental techniques}

As mentioned earlier, the most important characteristic of the aerosol produced by nebulizers is the droplet size distribution (DSD). Since aerosol concentration in the emitted cloud is very high, the application of typical methods of aerosol characterization (such as optical particle counting) is limited. High dilution of the nebulized mists with clean air required in the counters usually results in partial evaporation of droplets. Therefore, the recorded DSD shows significantly smaller droplets than initially emitted from the nebulizer. The evaporation as the effect of a high thermal capacity of the metal device also limits the use of cascade impactors, although they are typically recommended for the characterization of pharmaceutical aerosols. Some researchers suggested that impactors should be cooled to eliminate the problem, but it makes the measurements more problematic and time-consuming.

From the above, it seems that laser diffraction offers the most convenient method of DSD determination. The optical signal is scattered on the whole concentrated aerosol cloud, which can be measured directly at the nebulizer outflow (i.e., without dilution). Either the Fraunhofer or Mie theory allows determining the volume-based DSD, taking into account the refraction index of droplets and the gas phase (air). The method available in our lab uses the Spraytec ${ }^{\circledR}$ spectrometer (Malvern, UK). The atomization output rate can be obtained from the gravimetric analysis, i.e., from weighing the nebulizing vessel at the given time intervals. The essential physicochemical properties of atomized liquids are determined in our studies as follows:

a) rheologic characteristics: MCR 102 rheometer (Anton Paar, Austria),

b) the surface tension (equilibrium/dynamic values): pendant/oscillating drop tensiometer PAT-1M (Sinterface, Germany),

c) zeta-potential and crystal size (in liquid suspensions): Nanosizer ZS (Malvern, UK),

d) liquid suspension stability: Genesys 10S spectrophotometer (Thermo Scientific, USA).

Aerosol velocity profile at the nebulizer outflow can be measured by several methods, including particle image velocimetry (PIV) or laser doppler anemometry (LDA). Both techniques require sophisticated equipment, so they are not often used for nebulizer characterization. In our laboratory, we have an opportunity to use 2D LDA system (Dantec, Denmark) for this purpose [1].

\section{Conclusion}

In this communication, we indicate selected technical challenges during the assessment of the atomization process in medical nebulizers. The physiochemical characteristics of liquids and the specific properties of the nebulizing device should be analyzed simultaneously to obtain crucial information on the therapeutic potential of the emitted aerosol. We suggest that chemical engineering offers valuable scientific tools to optimize nebulizer use in clinical practice.

\section{Acknowledgments}

Work done within the NCN project No. 2018/29/B/ST8/00273.

\section{References}

[1] Dobrowolska, K., Sosnowski, T. R. (2020). Evolution of droplet size distribution in selected nebulizers. Physicochem. Probl. Miner. Processing 56(6), 32-40. https://doi.org/ $10.37190 / \mathrm{ppmp} / 126312$. 